\title{
Protocols to test the activity of antimicrobial peptides against the honey bee pathogen Paenibacillus larvae
}

\author{
Jasmin C. Khilnani ${ }^{1 *}$ and Helen J. Wing ${ }^{1 \S}$ \\ ${ }^{1}$ School of Life Sciences, University of Nevada, Las Vegas, Las Vegas, NV 89154-4004 \\ ${ }^{\S}$ Corresponding author
}

Email addresses:

JCK: smithi23@unlv.nevada.edu

§HJW: helen.wing@unlv.edu Tel: 0017028955382

Mailing Address:

Helen J Wing

4505 South Maryland Parkway, Bldg WHI, M/S 4004

School of Life Sciences,

University of Nevada, Las Vegas,

Las Vegas, NV 89154-4004

Keywords: Paenibacillus larvae, American Foulbrood disease, antimicrobial compounds, honey bee 


\section{Abstract}

Paenibacillus larvae is the causal agent of the honey bee disease American foulbrood. Two enhanced protocols that allow the activity of antimicrobial peptides to be tested against $P$. larvae are presented. Proof of principle experiments demonstrate that the honey bee antimicrobial peptide Defensin 1 is active in both assays. 
American Foulbrood disease (AFB) is a contributing factor in the decline of honey bee (Apis mellifera) populations (Evans and Schwarz, 2011). It is the most destructive and widespread of all the bee brood diseases (Shimanuki, 1983). Infectious spores of $P$. larvae infect honey bee larvae within 72 hours of eclosion (hatching from the egg), but adults are resistant to AFB. Despite its name, AFB is found worldwide, wherever honey bees are kept (Matheson, 1993). While antibiotics can be used as effective prophylaxes, they are not recommended for the treatment of colonies that are actively infected, because this can i) select for resistant strains of P. larvae (Murray et al., 2007), ii) lead to traces of antibiotics in honey, and iii) trigger vegetative $P$. larvae cells to sporulate, where they remain as endospores until antibiotic levels decrease, at which time a new infection can occur (Genersch, 2010; Lindstrom et al., 2008). Consequently, there is need to improve upon the current strategies used to control this disease and so alternative treatment strategies and/or prophylaxes for AFB are currently being investigated (Alvarado et al., 2015; Alvarado et al., 2013; Beims et al., 2015; Chan et al., 2009; GhorbaniNezami et al., 2015; Gonzalez et al., 2015; Oliveira et al., 2015; Piana et al., 2015).

Antimicrobial peptides (AMPs) synthesized within the resistant adult honey bee provide an attractive solution to this problem because they have the potential to be used to fortify the natural immune system of the susceptible larvae. Furthermore, it has been proposed that bacteria are unlikely to develop resistance to these AMPs, because these natural compounds will not present a new selection pressure for these bacteria (Boman, 2003). Although methods to measure the activity of antimicrobials on P. larvae exist (de Graaf et al., 2013), these protocols require relatively large quantities of the antimicrobial and are not optimized for larger molecular weight $(>500 \mathrm{Da}$ ) molecules like AMPs. Instead they were developed to test the resistance/susceptibility of $P$. larvae to commercially available antibiotics (de Graaf et al., 2013).

Here, we present and compare two newly enhanced protocols that allow the antimicrobial activity of low abundance, high molecular weight compounds, like AMPs, to be tested against $P$. larvae in semi-solid and liquid media. The first protocol is a modified zone of inhibition assay, in 
which test compounds are applied directly to a well in a low percentage agar plate. The second protocol is an adaptation of a minimal inhibitory concentration (MIC) assay, in which the antimicrobial activity of compounds is tested in 96-well plate format. The advantage of both of these protocols over currently published methods is that they allow the antimicrobial activity of candidate compounds that are high in molecular weight, low in supply or otherwise prohibitively expensive to be tested.

In this study, our newly adapted methods were used to test the activity of two active honey bee AMPs against either $P$. larvae strain B-3554 (American Tissue Culture Collection number; NRRL B-3554) or E. coli MC4100. For each of our growth inhibition assays, cultures were grown from frozen glycerol stocks (20\% v/v glycerol) in R2B medium (EMD Millipore) at 3x concentration (3x R2B). This medium was chosen for these studies because i) $P$. larvae grows well in this medium, ii) it is commercially available, iii) it has similar composition to MYPGP, a medium used for this kind of assay previously (de Graaf et al., 2013), and iv) unlike other commonly used broths, such as Brain Heart Infusion (BHI) medium, it does not contain sodium chloride, which has been shown to inhibit the function of honey bee AMPs (Casteels et al., 1989; Casteels et al., 1990; Chi et al., 2003). Cultures were routinely grown for $16 \mathrm{~h}$ at $37^{\circ} \mathrm{C}$ with aeration in an orbital shaker set to $325 \mathrm{rpm}$ (MaxQ 4000 Thermo Scientific), sub-cultured $(1: 20)$ and grown for an additional $16 \mathrm{~h}$ at $37^{\circ} \mathrm{C}$ with aeration. This method of growth was found to minimize $P$. larvae cell aggregation, which became important for our 96 -well plate assays.

For our modified zone of inhibition assays, $500 \mu \mathrm{l}$ of $P$. larvae and $250 \mu \mathrm{l}$ of $E$. coli overnight culture were harvested and pelleted by centrifugation. The supernatant was discarded, cells were resuspended in $150 \mu \mathrm{l}$ of fresh $3 x \mathrm{R} 2 \mathrm{~B}$, and cell suspensions were spread onto 3x R2A plates containing $1.5 \%(\mathrm{w} / \mathrm{v})$ agar (reduced from the standard $2 \%)$. These inocula ultimately allowed the growth of robust bacterial lawns. Once the cell suspension had been absorbed, a hole was punched into the center of the agar using a sterile, pre-cut pipet tip, and $2 \mu \mathrm{l}$ of the 
AMPs at a concentration $4 \mathrm{mg} \mathrm{ml}^{-1}$ (solubilized per manufacturer's instructions) was added to the hole. Plates were sealed with Parafilm ${ }^{\circledR}$ to prevent dehydration and incubated at $37^{\circ} \mathrm{C}$. Zones of inhibition were measured after $48 \mathrm{~h}$. All experiments were done in triplicate.

For our 96-well plate assays, overnight cultures of $P$. larvae and E. coli cultures were diluted in $3 x$ R2B medium to an $\mathrm{OD}_{600}$ of about 0.2 . The inner wells of a clear, flat-bottomed, 96 -well plate (Greiner Bio-One) containing $5 \mu$ l of AMP (ranging from 200 to $0.2 \mu \mathrm{g} \mathrm{ml}^{-1}$ ) or distilled water were filled with $95 \mu \mathrm{l}$ of inoculum. To minimize evaporation and other edge effects, outermost wells of plates were filled with $100 \mu$ l of water. Plates were incubated for $24 \mathrm{~h}$ at $37^{\circ} \mathrm{C}$ in a Tecan m200 plate reader, which shook the plate in orbital mode at $336 \mathrm{rpm}$ for 5 min every $15 \mathrm{~min}$ (amplitude of $1.5 \mathrm{~mm}$ ). Five absorbance scans at $600 \mathrm{~nm}$ were taken every 15 minutes and the average was reported. Growth curves were plotted to determine whether the AMP affected cell growth by comparing the antimicrobial-treated samples to water-treated controls. On each plate five replicate wells were used per condition and all experiments were independently replicated three times.

Using these approaches, two AMPs produced by adult honey bees, the bacteriostatic compound apidaecin (Anaspec; Fremont, CA; MW 2.1 KDa) and the bactericidal compound defensin 1 (Peptide 2.0, Chantilly, VA; MW 5.5 KDa), were tested against $P$. larvae and $E$. coli [E. coli was previously demonstrated to be sensitive to apidaecin in liquid assays (Casteels et al., 1989) while defensin 1 was previously shown to be active against $P$. larvae in a polyacrylamide gel overlay experiment (Bachanova et al., 2002)]. As expected, apidaecin exhibited detectable antibacterial activity against $E$. coli in both the zone of inhibition assay (8 $\mathrm{mm}$ in diameter; Figure $1 \mathrm{~A}$ ) and the 96 -well plate assay over the $24 \mathrm{~h}$ incubation period (Figure 1E). In contrast, defensin 1 did not display antimicrobial activity against $E$. coli in either assay (Figure 1, C \& G), which is consistent with previous findings that demonstrate this AMP is primarily active against Gram-positive bacteria (Ilyasov et al., 2013). When the antimicrobial 
activity of apidaecin was measured against $P$. larvae, no growth inhibition was detected in the zone of inhibition assays (Figure 1B) but surprisingly certain concentrations displayed modest but significant antibacterial activity against $P$. larvae in the 96 -well plate assay over the $24 \mathrm{~h}$ incubation period (Figure 1F). In contrast, as predicted (Bachanova et al., 2002) defensin 1 displayed robust antimicrobial activity against $P$. larvae in both the zone of inhibition $(6 \mathrm{~mm}$ in diameter; Figure 1D) and the 96-well plate assay (Figure 1H).

To conclude, this study demonstrates that two newly adapted protocols allow the antimicrobial activity of high molecular weight, low abundance compounds, like AMPs, to be tested against $P$. larvae. Each of the methods provide significant improvements over currently existing protocols [described in (de Graaf et al., 2013)], which were initially developed to test the resistance/susceptibility of $P$. larvae to commercially available antibiotics. In the zone of inhibition assay, by placing the AMP in a hole in the center of the agar plate, reducing the agar concentration and removing sodium chloride from the growth medium, the activity and diffusion of the AMPs through the agar was less impeded. As a result, for the first time apidaecin was found to be active against $E$. coli in semi-solid medium, which was not the case when we initially used the disc diffusion assay (de Graaf et al., 2013) to test the antimicrobial activity of this compound (data not shown). This demonstrates the newly adapted zone of inhibition assay is an improvement over the currently published disc diffusion assay (de Graaf et al., 2013). In the 96-well plate assay, by decreasing the volume of $P$. larvae cultures to $100 \mu \mathrm{l}$, much less test compound was required, making these assays much more suitable for testing the activity of low abundance compounds than the published MIC assays (de Graaf et al., 2013). For the compounds tested in this study, the 96-well plate assay proved to be more sensitive than the zone of inhibition assay, allowing even the modest antimicrobial activity of apidaecin to be detected against $P$. larvae (compare Figure $1 \mathrm{~F}$ to $1 \mathrm{~B}$ ). Nevertheless, we strongly recommend the use of both protocols when initially characterizing the antimicrobial activity of compounds like AMPs because the activity of these compounds may vary in semi-solid and liquid media. In 
summary, the protocol adaptations and the recommendations presented in this work will prove useful to those evaluating high molecular weight (up to $5.5 \mathrm{KDa}$ ), low abundance antimicrobials for use in treatments and /or prophylaxis strategies for AFB. 

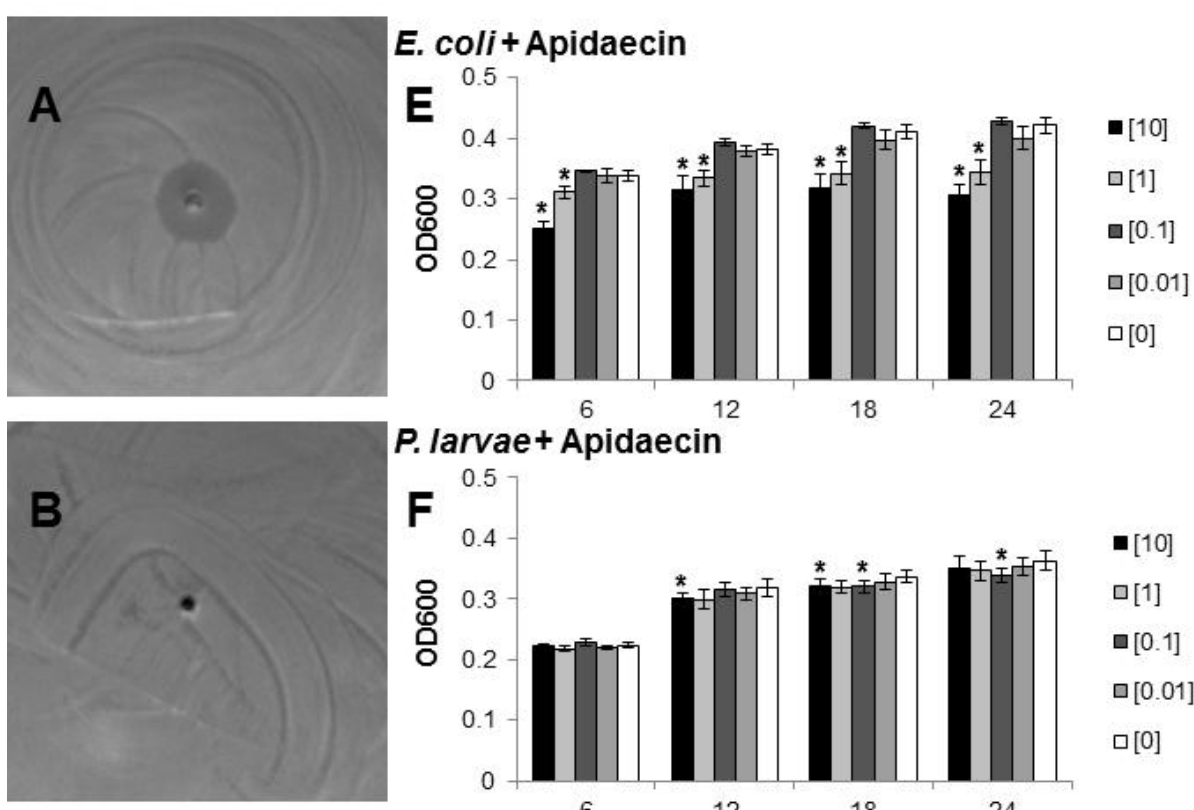

P. larvae+ Apidaecin
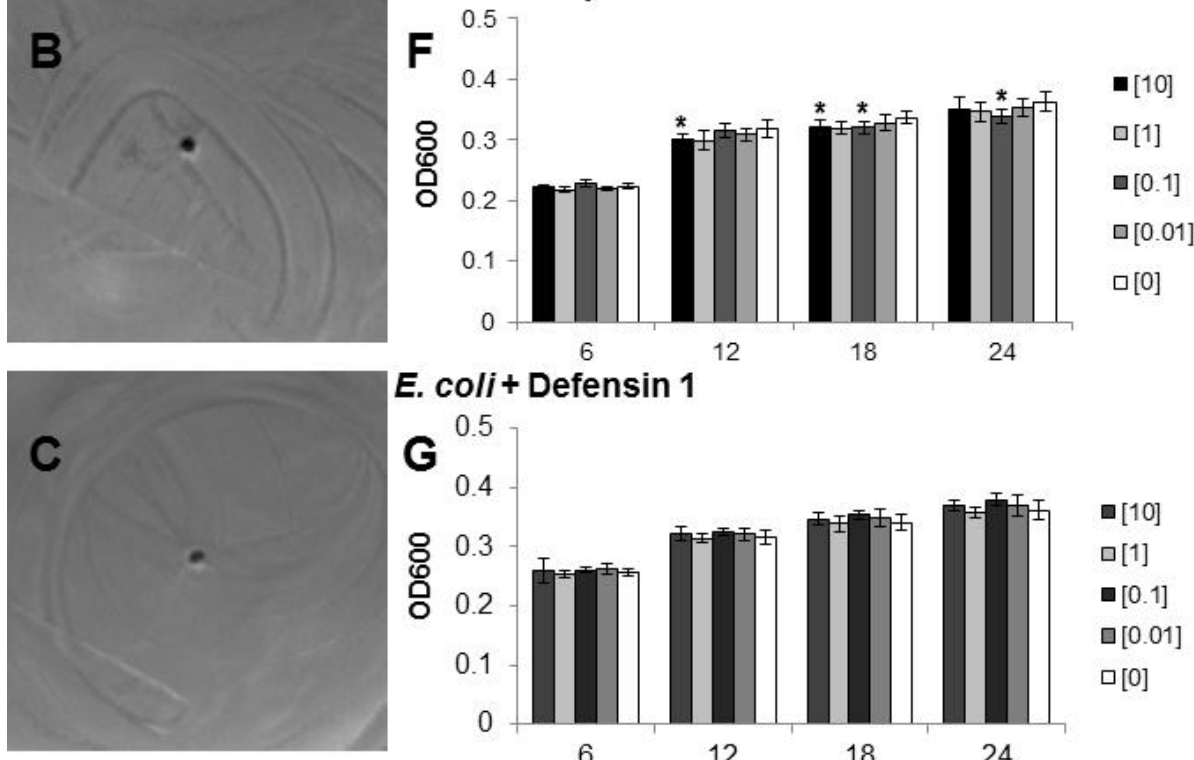

E. coli + Defensin 1
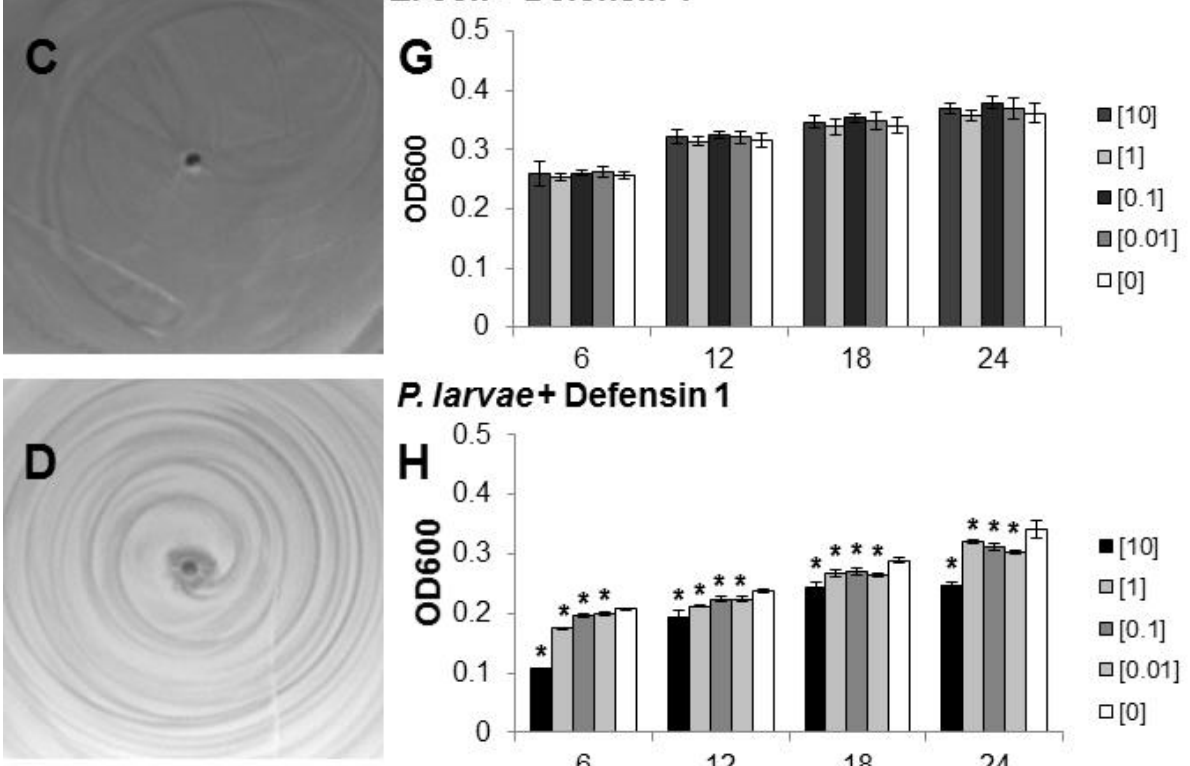

P. larvae+ Defensin 1

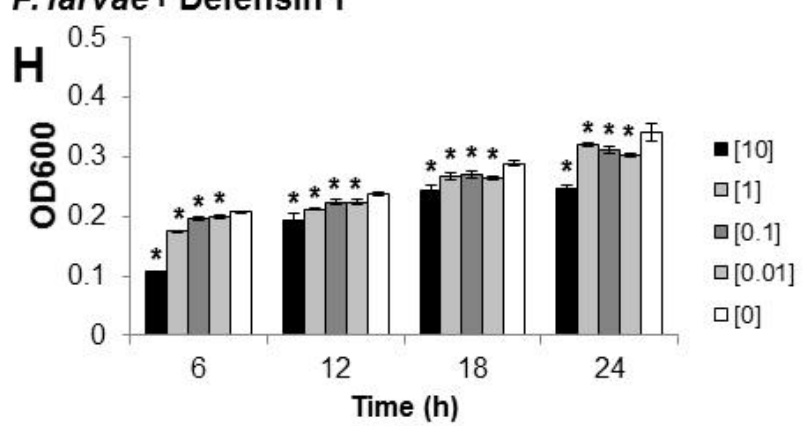

Figure 1 - Comparison of assays used to measure the antibacterial activity of honey bee AMPs. Results obtained after challenging bacterial strains with the honey bee AMPs apidaecin and defensin 1. Images of zone of inhibition assays (A-D) were taken after $48 \mathrm{~h}$ of growth. The $\mathrm{OD}_{600}$ of samples in the 96-well plate assays $(\mathrm{E}-\mathrm{H})$ were measured throughout a $24 \mathrm{~h}$ time course (all samples had an $\mathrm{OD}_{600}$ of 0.2 at time 0 , data not shown). Final concentrations of apidaecin and defensin 1 are shown $\left(\mu \mathrm{g} \mathrm{ml}^{-1}\right)$. Error bars represent standard deviations from the mean for the five replicate wells and statistical significance between each AMP-treated sample and the water-treated $(0)$ sample was determined using a Student's two-tailed $t$-test assuming equal variance. Stars $\left(^{*}\right)$ indicate $p$-values of $p \leq 0.05$. Data are representative of independent experiments done in triplicate. 


\section{Acknowledgements}

We thank M. Picker and Dr. N. Griffin for critical reading of the manuscript. This work was supported by USDA Grant NEVR-2010-03755.

\section{References}

Alvarado, I., Elekonich, M.M., Abel-Santos, E., and Wing, H.J. (2015). Comparison of in vitro methods for the production of Paenibacillus larvae endospores. Journal of microbiological methods 116, 30-32.

Alvarado, I., Phui, A., Elekonich, M.M., and Abel-Santos, E. (2013). Requirements for in vitro germination of Paenibacillus larvae spores. J Bacteriol 195, 1005-1011.

Bachanova, K., Klaudiny, J., Kopernicky, J., and Simuth, J. (2002). Identification of honeybee peptide active against Paenibacillus larvae larvae through bacterial growth-inhibition assay on polyacrylamide gel. Apidologie 33, 259-269.

Beims, H., Wittmann, J., Bunk, B., Sproer, C., Rohde, C., Gunther, G., Rohde, M., von der Ohe, W., and Steinert, M. (2015). Characterization of Paenibacillus larvae-directed bacteriophage HB10c2 and evaluation of its application in honey bee larvae suffering from American Foulbrood. Appl Environ Microbiol.

Boman, H.G. (2003). Antibacterial peptides: basic facts and emerging concepts. J Intern Med 254, 197-215.

Casteels, P., Ampe, C., Jacobs, F., Vaeck, M., and Tempst, P. (1989). Apidaecins -

Antibacterial Peptides from Honeybees. Embo J 8, 2387-2391.

Casteels, P., Ampe, C., Riviere, L., Vandamme, J., Elicone, C., Fleming, M., Jacobs, F., and

Tempst, P. (1990). Isolation and Characterization of Abaecin, a Major Antibacterial Response

Peptide in the Honeybee (Apis-Mellifera). Eur J Biochem 187, 381-386.

Chan, Q.W., Melathopoulos, A.P., Pernal, S.F., and Foster, L.J. (2009). The innate immune and systemic response in honey bees to a bacterial pathogen, Paenibacillus larvae. BMC Genomics 10, 387.

Chi, E.Y., Krishnan, S., Randolph, T.W., and Carpenter, J.F. (2003). Physical stability of proteins in aqueous solution: mechanism and driving forces in nonnative protein aggregation. Pharm Res 20, 1325-1336.

de Graaf, D.C., Alippi, A.M., Antunez, K., Aronstein, K.A., Budge, G., De Koker, D., De Smet, L., Dingman, D.W., Evans, J.D., Foster, L.J., et al. (2013). Standard methods for American foulbrood research. J Apicult Res 52.

Evans, J.D., and Schwarz, R.S. (2011). Bees brought to their knees: microbes affecting honey bee health. Trends Microbiol 19, 614-620.

Genersch, E. (2010). American Foulbrood in honeybees and its causative agent, Paenibacillus larvae. J Invertebr Pathol 103 Suppl 1, S10-19.

Ghorbani-Nezami, S., LeBlanc, L., Yost, D.G., and Amy, P.S. (2015). Phage Therapy is Effective in Protecting Honeybee Larvae from American Foulbrood Disease. Journal of insect science 15.

Gonzalez, M.J., Beoletto, V.G., Agnese, A.M., Audisio, M.C., and Marioli, J.M. (2015).

Purification of substances from Achyrocline satureioides with inhibitory activity against 
Paenibacillus larvae, the causal agent of American foulbrood in honeybees' larvae. Applied biochemistry and biotechnology 175, 3349-3359.

llyasov, R., Gaifullina, L., E, S., Poskryakov, A., and Nikolaenko, A. (2013). Defensins in the honeybee Antiinfectious Protection. Journal of Evolutionary Biochemistry and Physiology 49, 1-

9.

Lindstrom, A., Korpela, S., and Fries, I. (2008). The distribution of Paenibacillus larvae spores in adult bees and honey and larval mortality, following the addition of American foulbrood diseased brood or spore-contaminated honey in honey bee (Apis mellifera) colonies. J Invertebr Pathol 99, 82-86.

Matheson, A. (1993). World Bee Health Report. Bee World 74, 176-212.

Murray, K.D., Arotistein, K.A., and Leon, J.H. (2007). Analysis of pMA67, a predicted rollingcircle replicating, mobilizable, tetracycline-resistance plasmid from the honey bee pathogen, Paenibacillus larvae. Plasmid 58, 89-100.

Oliveira, A., Leite, M., Kluskens, L.D., Santos, S.B., Melo, L.D., and Azeredo, J. (2015). The First Paenibacillus larvae Bacteriophage Endolysin (PlyPI23) with High Potential to Control American Foulbrood. Plos One 10, e0132095.

Piana, M., Brum, T.F., Boligon, A.A., Alves, C.F., Freitas, R.B., Nunes, L.T., Mossmann, N.J., Janovik, V., Jesus, R.S., Vaucher, R.A., et al. (2015). In vitro growth-inhibitory effect of Brazilian plants extracts against Paenibacillus larvae and toxicity in bees. Anais da Academia Brasileira de Ciencias 87, 1041-1047.

Shimanuki, H. (1983). Identification and control of honey bee diseases, Vol 2255 (U.S. Dept. of agriculture). 